\title{
Atomization experiment of pulsed supersonic liquid jets
}

\author{
H.-H. Shi ${ }^{1}$, H. Sato ${ }^{2}$, and M. Itoh ${ }^{2}$ \\ 1 State Key Laboratory of Nonlinear Mechanics, Institute of Mechanics, \\ Chinese Academy of Sciences, 15 Beisihuanxi Road, Beijing 100080, P.R. China \\ 2 Department of Mechanical Engineering, Nagoya Institute of Technoiogy, Gcksso cho, \\ Showa-ku, Nagoya 466-8555, Japan
}

\begin{abstract}
This paper will int:odice an a, zomization txperinozt of pulsed supersonic water jets and polymer polyac-yianice (1:AA) ( $0.1 \%$ and $1.0 \%$ weight density) solution jets. The jets are generated from $n$ strall tigin-speed licuid jet apparatus. The schlieren photography is applied to visualize the jet:. 'The velocities of the jets are measured by cutting two laser beams. The effects of the nozzle diameter and the standoff distance on atomization and the jet velocity have been examined. The experiment shows that the polymer solution jets are easier to be atomized than water jets. This may be due to low surface tension of the polymer solution. The nozzle diameter causes different shock structures around the supersonic jets.
\end{abstract}

\section{Introduction}

The study of pulsed supersonic jets has wide applications in industrial and technological fields, such as water jet cutting, simulation of rain erosion of aircraft and wet steam turbines erosion, diesel fuel injection, road cleaning, etc. It also has important academic value because a supersonic liquid jet is a typical gasdynamic phenomenon. The jet generator can serve as a fundamental tool in gasdynamic research [1]. This paper shows an experimental study of pulsed supersonic water jets and PAA solution jets. One of the purposes of this paper is to examine the role of the polymer additive on injection and atomization of the jets.

\section{Experimental}

The supersonic liquid jets are generated from a small high-speed liquid jet apparatus. Figure 1 shows the jet generation method. A lead slug from an air rifle impacts the liquid, which is sealed in a stainless nozzle. The impact causes a high pressure in the nozzle, which will drive the liquid to flow through the nozzle exit to form a high-speed liquid jet. A schlieren optics is applied to visualize the jet and shock waves sequences. For the experimental details, one can see references of Shi and Itoh (1996), Shi and Sato (2004).

\section{Results}

\subsection{Jets from $0.5 \mathrm{~mm}$ nozzle}

Figure 2 shows the supersonic water jets from a $0.5 \mathrm{~mm}$ diameter nozzle. The photographs are taken by selecting different delay time to catch the injection sequences at different downstream distance. In Fig. 2(a), a thin jet ( $0.5 \mathrm{~mm}$ diameter) is followed by a large diameter of water. There are two distinguishable shock waves. One is the conical shock wave induced by the supersonic jet. Another is the spherical shock wave which is in front of the large diameter water. In Fig. 2(a), the jet has past the spherical wave. There are two questions that need to be clarified, that is, 


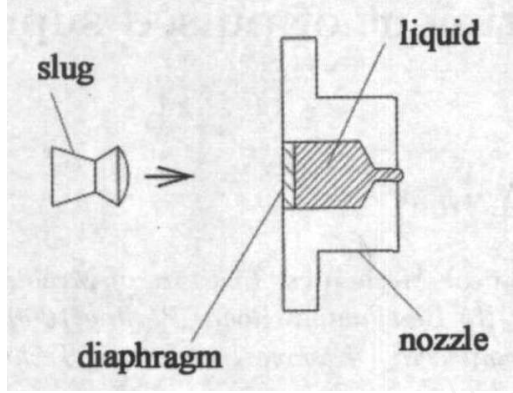

Fig. 1. Jet geiseration by the inpact of a lieas sligg

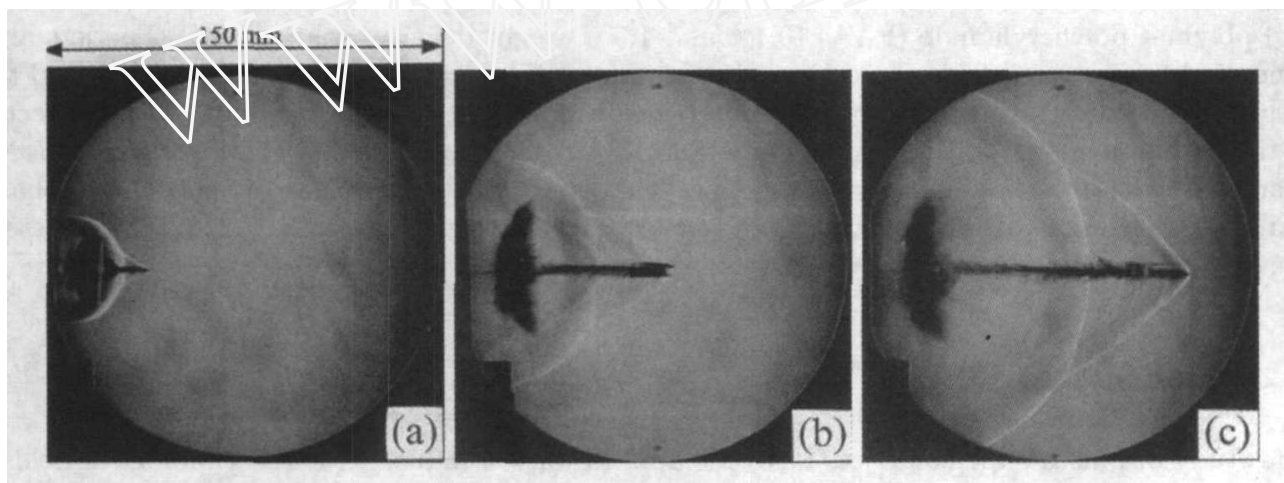

Fig. 2. Schlieren photographs of supersonic water jet from a $0.5 \mathrm{~mm}$ diameter nozzle

the mechanisms for the formation of the spherical shock wave and the formation of the large diameter water. Firstly, the spherical shock wave may be possibly a kind of blast wave. A blast wave can either be caused by a liquid shock wave passing through the liquid/air interface at the nozzle exit, or be caused by a sudden liquid motion at the straight section near the exit. However, in this experiment, the $0.5 \mathrm{~mm}$ straight section is very short (only $2 \mathrm{~mm}$ length) so that it is impossible to generate a shock wave with sufficient strength in this way. Meanwhile, it is because of the narrow channel of the $0.5 \mathrm{~mm}$ diameter straight section, it is also impossible for the transmitting shock wave to cause a explosive break-up of the liquid since it is found only with using $2 \mathrm{~mm}$ and $3 \mathrm{~mm}$ diameters nozzles (Shi and Takayama 1995, Shi et al. 2003a).

In Fig. 2(b), the large diameter water has become a disc-like jet near the nozzle exit. At later time shown in Fig. 2(c), the disc-like jet has lost its momentum while the jet head is traveling further downstream. The disc-like jet was once defined as a 'bifurcation' jet by Shi at al. (1993). Then it is re-defined as a 'spreading' jet by Shi and Sato (2004). It is seen from Figs. 2(b) and 2(c), the most velocity component of the spreading jet is in radial. It has been known that the explosive break-up of the liquid causes the jet has a most forward velocity component (Shi and Takayam 1995, Shi et al. 2003a). Now it is clear that the spreading jet is caused by the jet discontinuity due to the multiple liquid shock wave reflection in the nozzle (Shi and Itoh (1999). The spherical shock wave is not a blast wave but is induced by a rapid radial expansion of the spreading jet. It is also confirmed that the liquid shock wave is reflected back from the front wall although the nozzle exit is connected with the front wall.

In Fig. 2(a), the half angle of the Mach cone is about $\alpha=35$ degrees, so that the jet velocity is estimated as $V_{j}=\mathrm{a} / \mathrm{sin} \alpha=601 \mathrm{~m} / \mathrm{s}$, if the acoustic velocity in air is a $=345 \mathrm{~m} / \mathrm{s}$. The Mach cone angle increases in Figs. 2(b) and 2(c). This means the jet velocity decreases as the 
jet travels downstream. It is seen from Fig. 2, not only the leading shock wave interacts with the spherical wave, but also the Mach waves interact with the spherical wave. The bifurcation of the jet tip in Fig. 2(b) is associated with the Rayleigh-Taylor instability (Simpkins and Bales 1972). The rough boundary of the jet shown in Fig. 2(c) is due to the Kelvin-Helmholtz instability (Shi et al. 2001), which is one of the major reasons of causing liquid atomization.

\subsection{Jets from $2 \mathrm{~mm}$ nozzle}

Figure 3 shows supersonic water jets from a $2 \mathrm{~mm}$ diameter nozzle. in Fig. 3(c), the 'ion'i angle of the Mach cone is about 55 degrees, so that the jet velocity is estimated $8 \mathrm{~s} 421 \mathrm{Ia} / \mathrm{s}$. This value is an approximate one because the shock wave is a detailie! shoir wave and the jer fronit shape is usually irregular. Therefore, thera is sorne srror to deternine the jot velocity directly in this way (Shi et al. 2003b).
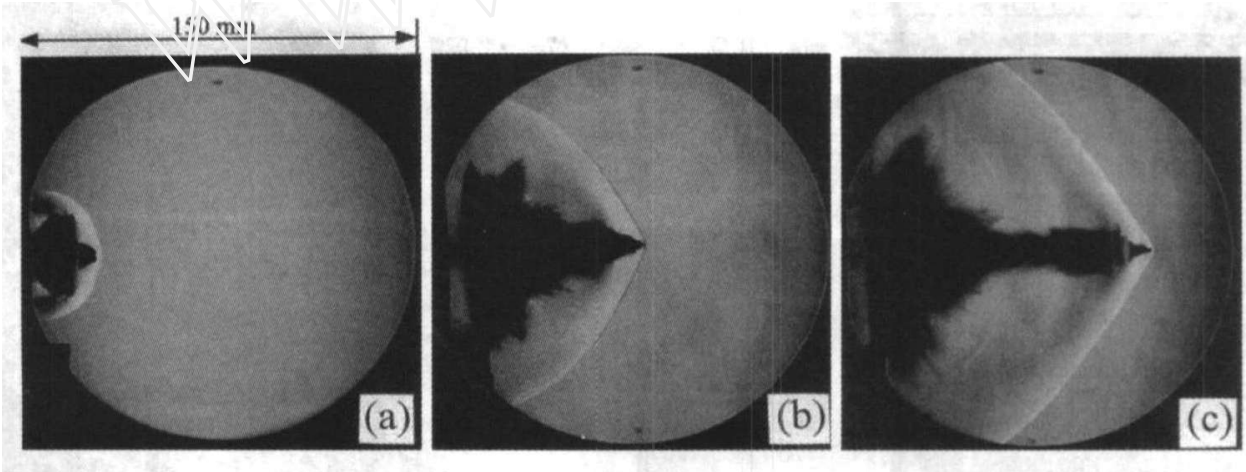

Fig. 3. Schlieren photographs of supersonic water jet from a $0.5 \mathrm{~mm}$ diameter nozzle

Similar to the $0.5 \mathrm{~mm}$ jet, the $2 \mathrm{~mm}$ jet also consists of two parts of liquid which have different moving directions, that is, the forward moving jet head and the radially moving spreading jet. In Fig. 3(a), a round jet head has emerged from the water disc. In Figs. 3(b) and 3(c), the jet head becomes thinner and the leading shock wave and spherical shock wave have combined into one bow shock.

Figure 4 shows supersonic PAA solution jets ( $0.1 \%$ density) from a $2 \mathrm{~mm}$ diameter nozzle. From Figs. 4(a) and 3(a), it is seen that at about same standoff distance, the polymer solution jet has a thinner jet head. Comparing Figs. 4(b) and 4(c), it is found that the polymer solution jet appears serious discontinuity. Beside the radial spreading jet, there is a large diameter spray $\mathrm{B}$ and its induced shock wave A. Fig. 4(c) shows a large amount of liquid has been atomized. Each part of the jet generates a shock wave to form a much complicated shock wave system. This means that the jet is in disintegrating. The reason for this may be related to the low surface tension of the solution. The polymer additives are usually effective in drag reduction in internal flows. This experiment shows that they also enhance liquid atomization.

Figure 5 shows supersonic PAA solution jets ( $1 \%$ density) from a $2 \mathrm{~mm}$ diameter nozzle. In this case, the radial spreading jet becomes a well-formed liquid disc. In Fig. 5(b), A marks the disc and $\mathrm{B}$ marks the induced shock wave. In Fig. 5(c), the disc, which has a quite large amount of the liquid, is disintegrated. However, the type of the liquid break-up in Fig. 5 is different from those in Figs. 2-4. The liquid break-up in Fig. 5(c) cause many liquid ligaments that are not spray. It is believed that when the PAA density is increased to $1 \%$, the increased liquid viscosity or the appearance of visco-elasticity plays a role. 


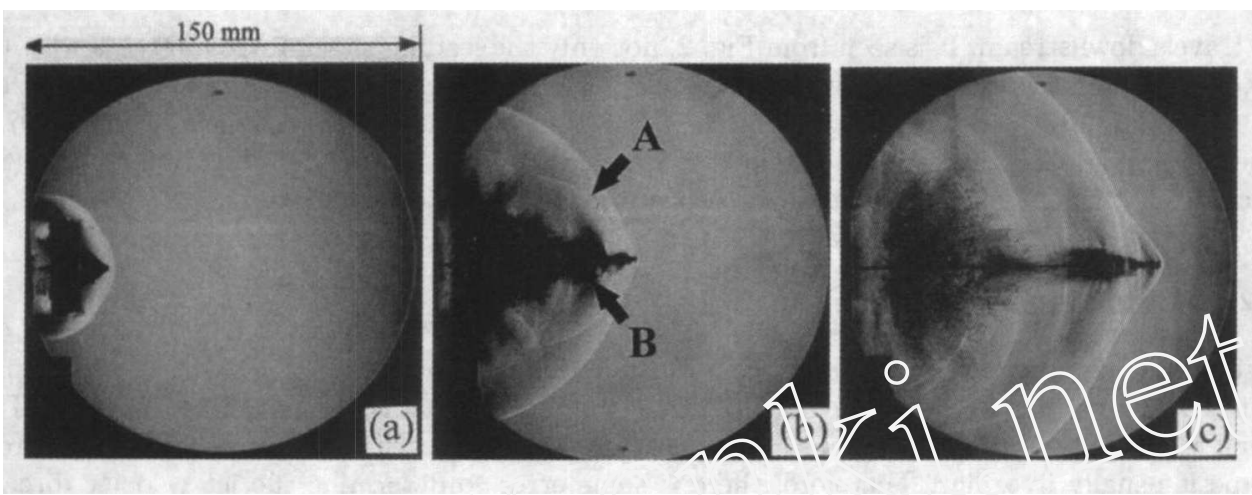

Fig. 4. Schlieren photcigraphs of supersonic v/ater jct̂ nom a $0.5 \mathrm{~mm}$ diameter nozzle
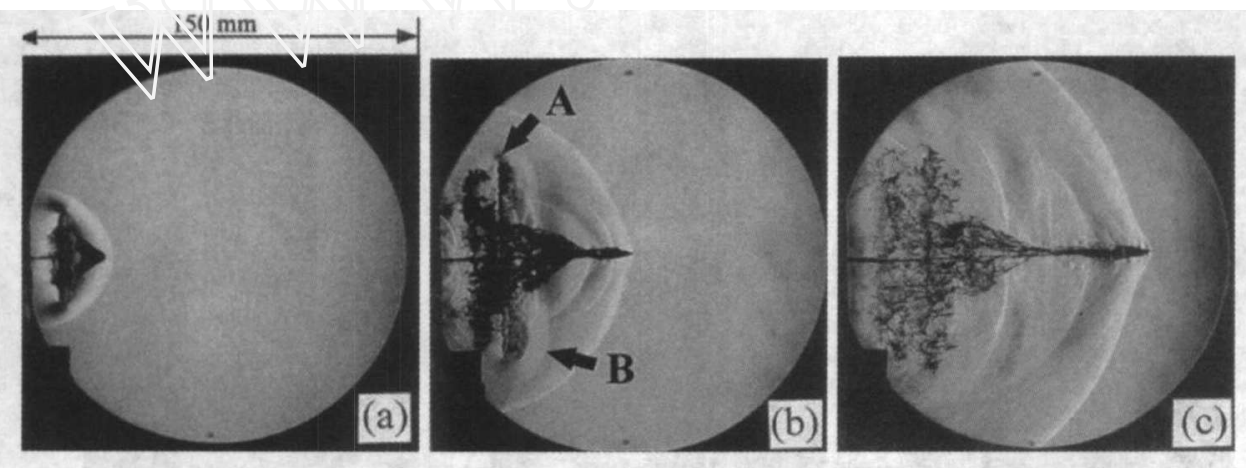

Fig. 5. Schlieren photographs of supersonic water jet from a $0.5 \mathrm{~mm}$ diameter nozzle

\section{Concluding remarks}

With using the jet generation method shown in Fig. 1, the jet has two main parts which have different moving directions. One is forward moving jet head following by a long tail. Another is radial moving spreading jet. The jet head induces a bow shock and the jet tail induces Mach waves. The spreading jet induced a spherical shock wave. With adding polymer additives into water, the spreading jet can become a well formed liquid disc and its break-up atomizes most of the liquid. The polymer additive causes the jet to be easily disintegrated so that resulting a much complicated shock wave system. The amount of the polymer additive plays a significant role in liquid atomization, e.g., liquid viscosity (or visco-elasticity) may influence the atomization process.

\section{References}

1. H.-H Shi, K. Takayama, O. Onodera: Experimental study of pulsed high-speed jet. JSME Int J Ser B 36, 620 (1993)

2. H.-H Shi, K. Takayama: Generation of high-speed liquid jets by high-speed impact of a projectile. JSME Int J Ser B 38(2), 181 (1995)

3. H.-H Shi, M. Itoh: Design and experiment of a small high-speed liquid jet apparatus. Jpn J Appl Phys 35(7), 327 (1996)

4. H.-H Shi, M. Itoh: Generation of high-speed liquid jet from a rectangular nozzle. Trans Japan Soc Aeron Space Sci 41: 195 (1999) 
5. H.-H Shi, K. Takayama: Generation of hypersonic liquid fuel jets accompanying selfcombustion. Shock Waves 9: 327 (1999)

6. H.-H Shi, X.L. Wang, M. Itoh, K. Koshiyama, Z. Huang: Unsteady liquid jet flowing through a rectangular nozzle. Exp Meas Fluid Mech 15(2): 59 (2001)

7. H.-H Shi, T. Hashiura, B.E. Milton: Evaluation of the cumulation formation of high-speed liquid jets. Journal of Hydrodynamics Ser B 15(3): 57 (2003)

8. H.-H Shi, T.J. Wang, B.E. Milton: Aerodynamic characteristics of supersonic liquid jets. Acta Aerodynamica Sinica (in Chinese) (in review) (2003)

9. H.-H Shi, H. Sato: Comparison between the impact extrusion and cumulation methoris in the generation of high-speed liquid jets. Experiments in Filids (in publicrion) (\%2s)

10. P.G. Simpkins, E.L. Bales: Water-drop response to sudden axcelertition. I Fluid Mer!: BS: $629(1972)$ 